\title{
On Topological Properties of Wireless Sensor Networks under the $q$-Composite Key Predistribution Scheme with On/Off Channels
}

\author{
Jun Zhao \\ CyLab and Dept. of ECE \\ Carnegie Mellon University \\ Pittsburgh, PA 15213 \\ Email: junzhao@junzhao.info
}

\author{
Osman Yağan \\ CyLab and Dept. of ECE \\ Carnegie Mellon University \\ Moffett Field, CA 94035 \\ Email: oyagan@ece.cmu.edu
}

\author{
Virgil Gligor \\ CyLab and Dept. of ECE \\ Carnegie Mellon University \\ Pittsburgh, PA 15213 \\ Email: gligor@cmu.edu
}

\begin{abstract}
The $q$-composite key predistribution scheme [1] is used prevalently for secure communications in large-scale wireless sensor networks (WSNs). Prior work [2]-[4] explores topological properties of WSNs employing the $q$-composite scheme for $q=1$ with unreliable communication links modeled as independent on/off channels. In this paper, we investigate topological properties related to the node degree in WSNs operating under the $q$-composite scheme and the on/off channel model. Our results apply to general $q$ and are stronger than those reported for the node degree in prior work even for the case of $q$ being 1 . Specifically, we show that the number of nodes with certain degree is asymptotically equivalent in distribution to a Poisson random variable, present the asymptotic probability distribution for the minimum degree of the network, and establish the asymptotically exact probability for the property that the minimum degree is at least an arbitrary value. Numerical experiments confirm the validity of our analytical findings.

Index Terms-Random intersection graph, random key graph, s-intersection graph, connectivity, node degree, key predistribution, wireless sensor network.
\end{abstract}

\section{INTRODUCTION}

The basic key predistribution scheme of Eschenauer and Gligor [5] has been recognized as a typical solution to secure communication in wireless sensor networks (WSNs) and studied extensively in the literature over the last decade [1][11]. The idea is that cryptographic keys are assigned before deployment to ensure secure sensor-to-sensor communications.

The $q$-composite key predistribution scheme proposed by Chan et al. [1] as an extension of the basic Eschenauer-Gligor scheme [5] (the $q$-composite scheme in the case of $q=1$ ) has received much interest [7], [18]-[23] since its introduction.

The $q$-composite scheme works as follows. For a WSN with $n$ sensors, prior to deployment, each sensor is independently assigned $K_{n}$ different keys which are selected uniformly at random from a pool of $P_{n}$ keys. Then two sensors establish a secure link in between after deployment if and only if they share at least $q$ key(s) and the physical link constraint between them is satisfied. $P_{n}$ and $K_{n}$ are both functions of $n$ for generality, with the natural condition $1 \leq K_{n} \leq P_{n}$. Examples of physical link constraints include the reliability of the transmission channel and the distance between two sensors close enough for communication. The $q$-composite scheme with $q \geq 2$ outperforms the basic Eschenauer-Gligor scheme with $q=1$ in terms of the strength against small-scale network capture attacks while trading off increased vulnerability in the face of large-scale attacks [1].
In this paper, we investigate topological properties related to node degree in WSNs employing the $q$-composite key predistribution scheme with general $q$ under the on/off channel model as the physical link constraint comprising independent channels which are either on or off. The degree of a node $v$ is the number of nodes having secure links with $v$; and the minimum (node) degree of a network is the least among the degrees of all nodes. Specifically, we demonstrate that the number of nodes with certain degree is asymptotically equivalent in distribution to a Poisson random variable, establish the asymptotic probability distribution for the minimum degree of the network, and derive the asymptotically exact probability for the property that the minimum degree is no less than an arbitrary value. Yağan [2] and we [3], [4] consider the WSNs with $q=1$ and show results for several topological properties, yet results about node degree in these prior work are weaker than our analytical findings even when the general $q$ is set as 1.

Our approach to the analysis is to explore the induced random graph models of the WSNs. As will be clear in Section [II, the graph modeling a WSN under the $q$-composite scheme and the on/off channel model is an intersection of two distinct types of random graphs. It is the intertwining [2], [34], [36], [39] of these two graphs that makes our analysis challenging.

We organize the rest of the paper as follows. Section III describes the system model in detail. Afterwards, we present and discuss the results in Section [II] Subsequently, we provide numerical experiments in Section IV to confirm our analytical results, whereas Section $\nabla$ is devoted to relevant results in the literature. Next, we conclude the paper and identify future research directions in Section VI

\section{System Model}

We elaborate the graph modeling of a WSN with $n$ sensors, which employs the $q$-composite key predistribution scheme and works under the on/off channel model. We use a node set $\mathcal{V}=\left\{v_{1}, v_{2}, \ldots, v_{n}\right\}$ to represent the $n$ sensors. For each node $v_{i} \in \mathcal{V}$, the set of its $K_{n}$ different keys is denoted by $S_{i}$, which is uniformly distributed among all $K_{n}$-size subsets of a key pool of $P_{n}$ keys.

\footnotetext{
${ }^{1} \mathrm{~A}$ sensor is also referred to as a node.
} 
The $q$-composite key predistribution scheme is modeled by a uniform $q$-intersection graph denoted by ${ }^{2} G_{q}\left(n, K_{n}, P_{n}\right)$, which is defined on the node set $\mathcal{V}$ such that any two distinct nodes $v_{i}$ and $v_{j}$ sharing at least $q$ key(s) (an event denoted by $\Gamma_{i j}$ ) have an edge in between. Clearly, $\Gamma_{i j}$ equals event $\left[\left|S_{i} \cap S_{j}\right| \geq q\right]$, where $|A|$ with $A$ as a set means the cardinality of $A$.

Under the on/off channel model, each node-to-node channel is independently on with probability $p_{n}$ and off with probability $\left(1-p_{n}\right)$, where $p_{n}$ is a function of $n$ with $0<p_{n} \leq 1$. Denoting by $C_{i j}$ the event that the channel between distinct nodes $v_{i}$ and $v_{j}$ is on, we have $\mathbb{P}\left[C_{i j}\right]=p_{n}$, where $\mathbb{P}[\mathcal{E}]$ denotes the probability that event $\mathcal{E}$ happens, throughout the paper. The on/off channel model is represented by an ErdősRényi graph $G\left(n, p_{n}\right)$ [52] defined on the node set $\mathcal{V}$ such that $v_{i}$ and $v_{j}$ have an edge in between if event $C_{i j}$ occurs.

Finally, we denote by $\mathbb{G}_{q}\left(n, K_{n}, P_{n}, p_{n}\right)$ the underlying graph of the $n$-node WSN operating under the $q$-composite key predistribution scheme and the on/off channel model. We often write $\mathbb{G}_{q}$ rather than $\mathbb{G}_{q}\left(n, K_{n}, P_{n}, p_{n}\right)$ for notation brevity. Graph $\mathbb{G}_{q}$ is defined on the node set $\mathcal{V}$ such that there exists an edge between nodes $v_{i}$ and $v_{j}$ if and only if events $\Gamma_{i j}$ and $C_{i j}$ happen at the same time. We set event $E_{i j}:=\Gamma_{i j} \cap C_{i j}$. Then $\mathbb{G}_{q}$ can be seen as the intersection of $G_{q}\left(n, K_{n}, P_{n}\right)$ and $G\left(n, p_{n}\right)$, i.e.,

$$
\mathbb{G}_{q}=G_{q}\left(n, K_{n}, P_{n}\right) \cap G\left(n, p_{n}\right) .
$$

We define $p_{s, q}$ as the probability that two different nodes share at least $q$ key(s) and $p_{e, q}$ as the probability that two distinct nodes have a secure link in $\mathbb{G}_{q}$. Clearly, $p_{s, q}$ and $p_{e, q}$ are the edge probabilities in graphs $G_{q}\left(n, K_{n}, P_{n}\right)$ and $\mathbb{G}_{q}$, respectively. $p_{s, q}$ and $p_{e, q}$ both depend on $K_{n}, P_{n}$ and $q$, while $p_{e, q}$ also depends on $p_{n}$. By definition, $p_{s, q}$ is determined through

$$
p_{s, q}=\mathbb{P}\left[\Gamma_{i j}\right]=\sum_{u=q}^{K_{n}} \mathbb{P}\left[\left|S_{i} \cap S_{j}\right|=u\right]
$$

where it is shown [6], [7] that

$$
\begin{aligned}
& \mathbb{P}\left[\left|S_{i} \cap S_{j}\right|=u\right] \\
& = \begin{cases}\frac{\left(\begin{array}{c}
K_{n} \\
u
\end{array}\right)\left(\begin{array}{c}
P_{n}-K_{n} \\
K_{n}-u
\end{array}\right)}{\left(\begin{array}{c}
P_{n} \\
K_{n}
\end{array}\right)}, & \text { for } \max \left\{0,2 K_{n}-P_{n}\right\} \leq u \leq K_{n}, \\
0, & \text { otherwise. }\end{cases}
\end{aligned}
$$

From $E_{i j}=\Gamma_{i j} \cap C_{i j}$ and the independence of $C_{i j}$ and $\Gamma_{i j}$, we obtain

$$
p_{e, q}=\mathbb{P}\left[E_{i j}\right]=\mathbb{P}\left[C_{i j}\right] \cdot \mathbb{P}\left[\Gamma_{i j}\right]=p_{n} \cdot p_{s, q} .
$$

\footnotetext{
${ }^{2}$ Many papers [7], [18]-[23] in the literature use $s$ instead of $q$ so we have uniform $s$-intersection graph $G_{s}\left(n, K_{n}, P_{n}\right)$. This work uses $q$ following the $q$-composite key predistribution scheme [1].
}

\section{THE RESULTS AND DISCUSSION}

We present and discuss our results in this section. Throughout the paper, $q$ is an arbitrary positive integer and does not scale with $n$; $e$ is the base of the natural logarithm function, ln. All limits are understood with $n \rightarrow \infty$. We use the standard asymptotic notation $o(\cdot), \omega(\cdot), O(\cdot), \Theta(\cdot), \sim$. In particular, for two sequences $f_{n}$ and $g_{n}, f_{n} \sim g_{n}$ signifies $\lim _{n \rightarrow \infty}\left(f_{n} / g_{n}\right)=1$; namely, $f_{n}$ and $g_{n}$ are asymptotically equivalent.

\section{A. Results of Graph $\mathbb{G}_{q}$}

We detail the results of graph $\mathbb{G}_{q}$ in Theorem 1 and Corollary 1 below. The detailed proofs of Theorem 1 and Corollary 1 can be found in the full version [6] and are omitted here owing to the space limitation. The basic idea is to use the method of moments [44].

Theorem 1. For graph $\mathbb{G}_{q}$ under $K_{n}=\omega(1)$ and $\frac{K_{n}^{2}}{P_{n}}=$ $o(1)$, the following properties (a) and (b) hold.

(a) If

$$
p_{e, q}=\Theta\left(\frac{\ln n}{n}\right),
$$

then for $h=0,1,2, \ldots$, it follows that $\phi_{h}$ denoting the number of nodes with degree $h$, is asymptotically equivalent in distribution to a Poisson random variable with mean $\lambda_{n, h}:=$ $n(h !)^{-1}\left(n p_{e, q}\right)^{h} e^{-n p_{e, q}}$. In other words, with $\operatorname{Po}\left(\lambda_{n, h}\right)$ denoting a Poisson random variable with mean $\lambda_{n, h}$, it holds that

$$
\mathbb{P}\left[\phi_{h}=i\right] \sim \mathbb{P}\left[\operatorname{Po}\left(\lambda_{n, h}\right)=i\right], \quad \text { for } i=0,1,2, \ldots
$$

(b) If for some integer $\ell$ and some sequence $\alpha_{n}$ satisfying

$$
-1<\liminf _{n \rightarrow \infty} \frac{\alpha_{n}}{\ln \ln n} \leq \limsup _{n \rightarrow \infty} \frac{\alpha_{n}}{\ln \ln n}<1,
$$

it holds that

$$
p_{e, q}=\frac{\ln n+(\ell-1) \ln \ln n+\alpha_{n}}{n},
$$

then defining $\delta$ as the minimum degree of $\mathbb{G}_{q}$, we obtain: for $\ell \leq 0$, it follows that as $n \rightarrow \infty$,

$$
\left\{\begin{array}{l}
\delta=0 \text { with a probability approaching to } 1, \\
\delta>0 \text { with a probability going to } 0
\end{array}\right.
$$

and for $\ell>0$, properties (b1)-(b4) below hold.

(b1) $(\delta \neq \ell) \cap(\delta \neq \ell-1)$ with a probability going to 0 as $n \rightarrow \infty$;

(b2) if $\lim _{n \rightarrow \infty} \alpha_{n}=\alpha^{*} \in(-\infty, \infty)$, then as $n \rightarrow \infty$,

$\left\{\begin{array}{l}\delta=\ell \text { with a probability converging to } e^{-\frac{e^{-\alpha^{*}}}{(k-1) !}} \\ \delta=\ell-1 \text { with a probability tending to }\left(1-e^{-\frac{e^{-\alpha^{*}}}{(k-1) !}}\right)\end{array}\right.$

(b3) if $\lim _{n \rightarrow \infty} \alpha_{n}=\infty$, then as $n \rightarrow \infty$,

$\left\{\begin{array}{l}\delta=\ell \text { with a probability approaching to } 1, \\ \delta \neq \ell \text { with a probability going to } 0 ;\end{array}\right.$ 
(b4) if $\lim _{n \rightarrow \infty} \alpha_{n}=-\infty$, then as $n \rightarrow \infty$,

$\left\{\begin{array}{l}\delta=\ell-1 \text { with a probability tending to } 1, \\ \delta \neq \ell-1 \text { with a probability converging to } 0 .\end{array}\right.$

Remark 1. Property (b) of Theorem 1 presents the asymptotic probability distribution for the minimum degree of the network.

We present a corollary of Theorem 1 below. The corollary is established with the help of a graph coupling argument [12] (see the full version [6] for the detailed proof).

Corollary 1. For graph $\mathbb{G}_{q}$ under $K_{n}=\omega(1)$ and $\frac{K_{n}{ }^{2}}{P_{n}}=$ $o(1)$, with some sequence $\beta_{n}$ defined by

$$
p_{e, q}=\frac{\ln n+(k-1) \ln \ln n+\beta_{n}}{n}
$$

for some positive integer $k$, and with $\delta$ denoting the minimum degree of $\mathbb{G}_{q}$, it holds that

$\lim _{n \rightarrow \infty} \mathbb{P}[\delta \geq k]= \begin{cases}e^{-\frac{e^{-\beta^{*}}}{(k-1) !},} & \text { if } \lim _{n \rightarrow \infty} \beta_{n}=\beta^{*} \in(-\infty, \infty), \\ 1, & \text { if } \lim _{n \rightarrow \infty} \beta_{n}=\infty, \\ 0, & \text { if } \lim _{n \rightarrow \infty} \beta_{n}=-\infty .\end{cases}$

Remark 2. Corollary 1 presents the asymptotically exact probability and a zero-one law [10] (a kind of phase transition [40]) for the event that graph $\mathbb{G}_{q}$ has a minimum node degree no less than $k$.

Remark 3. Setting $p_{n}$ to 1 in Theorem 1 and Corollary 1 we obtain corresponding results [24] for topological properties in uniform q-intersection graph $G_{q}\left(n, K_{n}, P_{n}\right)$.

Remark 4. In Theorem 1 and Corollary 1 given $K_{n}=\omega(1)$, we have $q<K_{n}$ for all $n$ sufficiently large since $q$ does not scale with $n$. From $\frac{K_{n}{ }^{2}}{P_{n}}=o(1)$, it is clear that $K_{n}<P_{n}$ for all $n$ sufficiently large.

\section{B. Practicality of Conditions}

We check the practicality of the conditions in Theorem 1 and Corollary 1) $K_{n}=\omega(1), \frac{K_{n}^{2}}{P_{n}}=o(1)$, and (4)-(6). First, the condition $K_{n}=\omega(1)$ follows in wireless sensor network applications [2] since $K_{n}$ is often logarithmic [2] with $n$, the number of sensor nodes in the network. Second, the condition $\frac{K_{n}{ }^{2}}{P_{n}}=o(1)$ also holds in practice since the key pool size $P_{n}$ is expected to be several orders of magnitude larger than $K_{n}$ [1], [5]. Finally, (4)- (6) present the range of $p_{e, q}$ that is of interest.

\section{Analogs of Theorem $\square$ and Corollary $\square$}

Analogous results to those of Theorem 1 and Corollary 1 can be given with $p_{e, q}$ at all places substituted by $p_{n}$. $\frac{1}{q !}\left(\frac{K_{n}^{2}}{P_{n}}\right)^{q}$, due to $p_{e, q}=p_{n} \cdot p_{s, q}$ from (3) and the replacement of $p_{s, q}$ by $\frac{1}{q !}\left(\frac{K_{n}^{2}}{P_{n}}\right)^{q}$ given Lemma 1 below. However, extra conditions have to be added for some results. The details as well as the proof of Lemma 1 are provided in the full version [6].

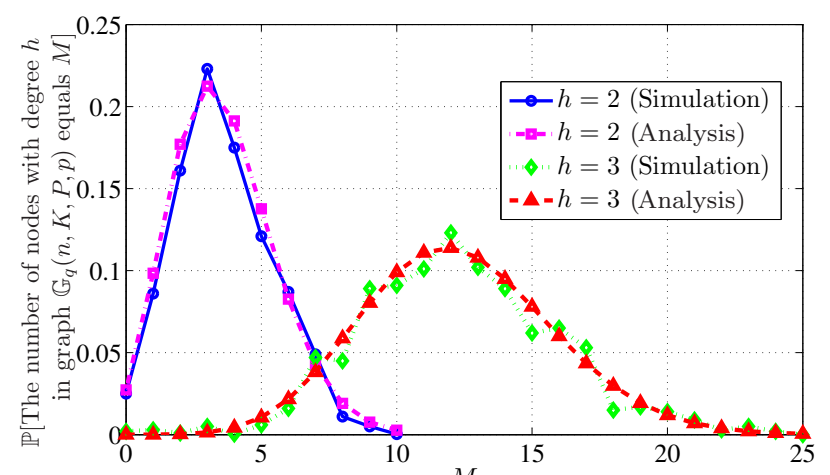

Fig. 1. A plot of the probability distribution for the number of nodes with degree $h$ for $h=2,3$ in graph $\mathbb{G}_{q}(n, K, P, p)$ with $n=2,000, q=2$, $P=10,000, K=36$ and $p=0.7$.

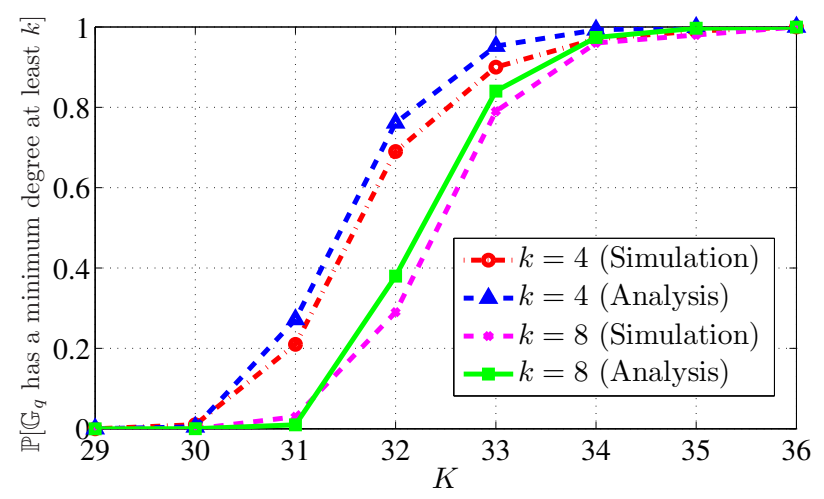

Fig. 2. A plot of the probability that graph $\mathbb{G}_{q}(n, K, P, p)$ has a minimum node degree at least $k$ as a function of $K$ for $k=4$ and $k=8$ with $q=2$, $n=2,000, P=10,000$, and $p=0.8$.

Lemma 1. If $K_{n}=\omega(1)$ and $\frac{K_{n}^{2}}{P_{n}}=o(1)$, then it follow that $p_{s, q} \sim \frac{1}{q !}\left(\frac{K_{n}^{2}}{P_{n}}\right)^{q}$.

\section{NUMERICAL EXPERIMENTS}

To confirm the results in Theorem 1, we now provide numerical experiments in the non-asymptotic regime. As we will see from the simulation results, the experimental observations are in agreement with our theoretical findings.

In all experiments, we fix the number of nodes at $n=2,000$ and the key pool size at $P=10,000$. In Figure 1 we plot the probability distribution for the number of nodes with degree $h$ in graph $\mathbb{G}_{q}(n, K, P, p)$ for $h=2,3$ from both the simulation and the analysis, with $q=2, K=36$ and $p=0.7$. On the one hand, for the simulation, we generate 2,000 independent samples of $\mathbb{G}_{q}(n, K, P, p)$ and record the count (out of a possible 2,000) that the number of nodes with degree $h$ for each $h$ equals a particular non-negative number $M$ ( $\mathrm{M}$ is the horizontal axis in Figure 1). Then the empirical probabilities are obtained by dividing the counts by 2,000 . On the other hand, we approximate the analytical curves by the asymptotic results as explained below. Property (a) of Theorem 1 notes that with the parameter conditions therein, the number of nodes in $\mathbb{G}_{q}\left(n, K_{n}, P_{n}, p_{n}\right)$ with degree $h$ is asymptotically equivalent in distribution to a Poisson random variable with mean $\lambda_{n, h}=n(h !)^{-1}\left(n p_{e, q}\right)^{h} e^{-n p_{e, q}}$. 
We derive $\lambda_{n, h}$ by computing the corresponding probability of $p_{e, q}$ in $\mathbb{G}_{q}(n, K, P, p)$ through

$$
p_{e, q}=p \cdot \sum_{u=q}^{K}\left[\left(\begin{array}{l}
K \\
u
\end{array}\right)\left(\begin{array}{l}
P-K \\
K-u
\end{array}\right) /\left(\begin{array}{l}
P \\
K
\end{array}\right)\right]
$$

given (13) and $P>2 K$. Then for each $h$, we plot a Poisson distribution with mean $\lambda_{n, h}$ as the curve corresponding to the analysis. We observe that the curves generated from the simulation and those obtained by the analysis are close to each other, confirming the result on asymptotic Poisson distribution in property (a) of Theorem 1 .

In Figure 2, we depict the probability that graph $\mathbb{G}_{q}(n, K, P, p)$ has a minimum node degree at least $k$ from both the simulation and the analysis, for $k=4$ and $k=8$ with $q=2$ and $p=0.8$ and $K$ varying from 29 to 36 (we still set $n=2,000$ and $P=10,000$ ). Similar to the experiments for Figure 1 above, we also generate 2,000 independent samples of graph $\mathbb{G}_{q}(n, K, P, p)$ and record the count that the minimum degree of graph $\mathbb{G}_{q}(n, K, P, p)$ is no less than $k$; and the empirical probability of $\mathbb{G}_{q}(n, K, P, p)$ having a minimum degree at least $k$ is derived by averaging over the 2,000 experiments. The analytical curves in Figure 2 are also approximated by the asymptotical results as follows. First, we compute the corresponding probability of $p_{e, q}$ in $\mathbb{G}_{q}(n, K, P, p)$ through (7). Then based on (6), we determine $\beta$ through $p_{e, q}=\frac{\ln n+(k-1) \ln \ln n+\beta}{n}$. Then with an approximation to the asymptotical results in Corollary 11 we plot the analytical curves by considering that the minimum degree of $\mathbb{G}_{q}(n, K, P, p)$ is at least $k$ with probability $e^{-\frac{e^{-\beta}}{(k-1) !}}$. The observation that the curves generated from the simulation and the analytical curves are close to each other is in accordance with Corollary 1

\section{RELATED WORK}

Erdôs and Rényi [52] propose the random graph model $G\left(n, p_{n}\right)$ defined on a node set with size $n$ such that an edge between any two nodes exists with probability $p_{n}$ independently of all other edges. For graph $G\left(n, p_{n}\right)$, Erdős and Rényi [52] derive the asymptotically exact probabilities for connectivity the property that the minimum degree is at least 1 , by proving first that the number of isolated nodes converges to a Poisson distribution as $n \rightarrow \infty$. Later, they extend the results to general $k$ in [53], obtaining the asymptotic Poisson distribution for the number of nodes with certain degree and the asymptotically exact probabilities for $k$-connectivity and the event that the minimum degree is at least $k$, where $k$ connectivity is defined as the property that the network remains connected in spite of the removal of any $(k-1)$ node 3 . Since its introduction, graph $G\left(n, p_{n}\right)$ has been widely investigated [25]-[35].

For graph $G_{q}\left(n, K_{n}, P_{n}\right)$, Bloznelis et al. [7] demonstrate that a connected component with at at least a constant fraction of $n$ emerges asymptotically when the edge probability $p_{e, q}$ exceeds $1 / n$. Bloznelis and Łuczak [21] have recently considered

\footnotetext{
${ }^{3} k$-connectivity throughout this paper means $k$-vertex-connectivity [51].
}

connectivity and perfect matching. Still in $G_{q}\left(n, K_{n}, P_{n}\right)$, Bloznelis et al. [20] investigate assortativity and clustering, while for asymptotic node degree distribution, Bloznelis [19] analyzes clustering coefficient and the degree distribution of a typical node. We [24] compute the probability distribution for the minimum node degree. Recently, Bloznelis and Rybarczyk [22] and we [23] have derived the asymptotically exact probability of $k$-connectivity. Several variants or generalizations of graph $G_{q}\left(n, K_{n}, P_{n}\right)$ are also considered in the literature [7], [18]-[20].

When $q=1$, for graph $G_{1}\left(n, K_{n}, P_{n}\right)$ (also referred to as a random key graph [8], [10], [11] or a uniform random intersection graph [9], [13]) and some of its variants, a number of properties have been extensively studied in the literature including component evolution [43], connectivity [9], [10], [13], $k$-connectivity [12], [37], node degree distribution [14][17] and independent sets [41], [42].

In graph $\mathbb{G}_{1}$, Yağan [2] presents zero-one laws for connectivity and for the property that the minimum degree is at least 1. We extend Yağan's results to general $k$ for $\mathbb{G}_{1}$ in [3], [4].

Krishnan et al. [8] and Krzywdziński and Rybarczyk [38] describe results for the probability of connectivity asymptotically converging to 1 in WSNs employing the $q$-composite key predistribution scheme with $q=1$ (i.e., the basic EschenauerGligor key predistribution scheme), not under the on/off channel model but under the well-known disk model [45][50], where nodes are distributed over a bounded region of a Euclidean plane, and two nodes have to be within a certain distance for communication. Simulation results in our work [3] indicate that for WSNs under the key predistribution scheme with $q=1$, when the on-off channel model is replaced by the disk model, the performances for $k$-connectivity and for the property that the minimum degree is at least $k$ do not change significantly.

\section{Conclusion and Future Directions}

In this paper, we analyze several topological properties related to node degree in a wireless sensor network operating under the $q$-composite key predistribution scheme with on/off channels. The network is modeled by the superposition of an Erdős-Rényi graph on a uniform $q$-intersection graph. Numerical simulation is shown to be in agreement with our theoretical findings.

Two future research directions are as follows. To begin with, we can consider physical link constraints different with the on/off channel model, where one candidate is the aforementioned disk model. Another extension is to derive the asymptotically exact probability and thus a zero-one law for $k$-connectivity in graph $\mathbb{G}_{q}$. Note that a zero-law for $k$ connectivity follows immediately from Corollary 1 since $k$ connectivity implies the property of minimum node degree being at least $k$. The one-law and the asymptotically exact probability result will follow if we show that under certain conditions, the probability that $\mathbb{G}_{q}$ has a minimum node degree no less than $k$ but is not $k$-connected converges to 0 as $n \rightarrow \infty$. 


\section{REFERENCES}

[1] H. Chan, A. Perrig, and D. Song. Random key predistribution schemes for sensor networks. In Proc. of IEEE Symposium on Security and Privacy, May 2003.

[2] O. Yağan. Performance of the Eschenauer-Gligor key distribution scheme under an on/off channel. IEEE Transactions on Information Theory, 58(6):3821-3835, June 2012.

[3] J. Zhao, O. Yağan, and V. Gligor. k-Connectivity in secure wireless sensor networks with physical link constraints - the on/off channel model. Arxiv e-prints, 2012. Available online at http://arxiv.org/abs/1206.1531

[4] J. Zhao, O. Yağan, and V. Gligor. Secure $k$-connectivity in wireless sensor networks under an on/off channel model. In Proc. of IEEE ISIT, pages 2790-2794, 2013.

[5] L. Eschenauer and V. Gligor. A key-management scheme for distributed sensor networks. In Proc. of ACM CCS, 2002.

[6] J. Zhao, O. Yağan, and V. Gligor. Topological properties of wireless sensor networks under the $q$-composite key predistribution scheme with unreliable links. Technical Report CMU-CyLab-14-002, CyLab, Carnegie Mellon University, January 2014. Available online at http://www.cylab.cmu.edu/files/pdfs/tech_reports/CMUCyLab14002.pdf

[7] M. Bloznelis, J. Jaworski, and K. Rybarczyk. Component evolution in a secure wireless sensor network. Netw., 53:19-26, January 2009.

[8] B. Krishnan, A. Ganesh, and D. Manjunath. On connectivity thresholds in superposition of random key graphs on random geometric graphs. In Proc. of IEEE ISIT, pages 2389-2393, 2013.

[9] K. Rybarczyk. Diameter, connectivity and phase transition of the uniform random intersection graph. Discrete Mathematics, 311, 2011.

[10] O. Yağan and A. M. Makowski. Zero-one laws for connectivity in random key graphs. IEEE Transactions on Information Theory, 58(5):2983-2999, May 2012.

[11] V. Gligor, A. Perrig, and J. Zhao. Brief encounters with a random key graph. In Proc. of Security Protocols Workshop (SPW) 2009. Lecture Notes in Computer Science (LNCS), volume 7028, 2013.

[12] K. Rybarczyk. Sharp threshold functions for the random intersection graph via a coupling method. Electr. Journal of Combinatorics, 18:3647, 2011.

[13] S. R. Blackburn and S. Gerke. Connectivity of the uniform random intersection graph. Discrete Mathematics, 309(16), August 2009.

[14] J. Jaworski, M. Karoński, and D. Stark. The degree of a typical vertex in generalized random intersection graph models. Discrete Mathematics, 306(18):2152 - 2165, 2006.

[15] M. Deijfen and W. Kets. Random intersection graphs with tunable degree distribution and clustering. Probability in the Engineering and Informational Sciences, 23:661-674, 2009.

[16] M. Bloznelis and J. Damarackas. Degree distribution of an inhomogeneous random intersection graph. The Electronic Journal of Combinatorics, 20(3):P3, 2013.

[17] D. Stark. The vertex degree distribution of random intersection graphs. Random Structures \& Algorithms, 24(3):249-258, 2004.

[18] M. Bradonjić, A. Hagberg, N. W. Hengartner, and A. G. Percus. Component evolution in general random intersection graphs. In $W A W$, pages 36-49, 2010

[19] M. Bloznelis. Degree and clustering coefficient in sparse random intersection graphs. The Annals of Applied Probability, 23(3):12541289, 2013.

[20] M. Bloznelis, J. Jaworski, and V. Kurauskas. Assortativity and clustering of sparse random intersection graphs. Electronic Journal of Probability, 18(38):1-24, 2013.

[21] M. Bloznelis and T. Łuczak. Perfect matchings in random intersection graphs. Acta Mathematica Hungarica, 138(1-2):15-33, 2013.

[22] M. Bloznelis and K. Rybarczyk. k-Connectivity of uniform $s$ intersection graphs. Discrete Mathematics, vol. 333, no. 0, pp. 94-100, 2014.

[23] J. Zhao, O. Yağan, and V. Gligor. On $k$-connectivity and minimum vertex degree in random $s$-intersection graphs. 2014. Available online at http://www.andrew.cmu.edu/user/junzhao/papers/s-int-k-con.pdf

[24] J. Zhao, O. Yağan, and V. Gligor. Results on vertex degree and $k$ connectivity in uniform $s$-intersection graphs. Technical Report CMUCyLab-14-004, CyLab, Carnegie Mellon University, January 2014. Available online at http://www.cylab.cmu.edu/files/pdfs/tech_reports/CMUCyLab14004.pdf
[25] E. Beer, J. A. Fill, S. Janson, and E. R. Scheinerman. On vertex, edge, and vertex-edge random graphs. The Electronic Journal of Combinatorics, 18(1):P110, 2011.

[26] P. Beame, J. Culberson, D. Mitchell, and C. Moore. The resolution complexity of random graph $k$-colorability. The Electronic Journal of Combinatorics, 153(1):25-47, 2005.

[27] A. Frieze and P. Loh. Rainbow hamilton cycles in random graphs. Random Structures \& Algorithms, 44(3): 328-354, 2014.

[28] A. Johansson, J. Kahn and V. Vu. Factors in random graphs. Random Structures \& Algorithms, 33(1): 1-28, 2008.

[29] F. Chung and L. Lu. The Diameter of Sparse Random Graphs. Advances in Applied Mathematics, 26(4): 257-279, 2001.

[30] A. Flaxman, A. Frieze, and J. Vera. Adversarial deletion in a scale free random graph process. In ACM SODA, pages 287-292, 2005

[31] A. Bonato, G. Hahn, and C. Wang. The Cop density of a graph. Contributions to Discrete Mathematics, 2(2): 133-144, 2007.

[32] D. Gamarnik and M. Sudan. Limits of local algorithms over sparse random graphs. In Innovations in Theoretical Computer Science conference (ITCS), 2014

[33] J. Ding, J. H. Kim, E. Lubetzky, and Y. Peres. Anatomy of a young giant component in the random graph. Random Structures \& Algorithms, 39(2):139-178, 2011.

[34] J. Zhao. Minimum node degree and k-connectivity in wireless networks with unreliable links. In Proc. of IEEE International Symposium on Information Theory (ISIT), 2014.

[35] B. Pittel and N. C. Wormald. Counting connected graphs inside-out. Journal of Combinatorial Theory, Series B, 93(2):127 - 172, 2005.

[36] M. Penrose. Connectivity of soft random geometric graphs. ArXiv eprints, 2013. Available online at http://arxiv.org/abs/1311.3897v1

[37] J. Zhao, O. Yağan, and V. Gligor. On the strengths of connectivity and robustness in general random intersection graphs. In IEEE Conference on Decision and Control (CDC), 2014.

[38] K. Krzywdziński and K. Rybarczyk. Geometric graphs with randomly deleted edges - connectivity and routing protocols. Mathematical Foundations of Computer Science, 6907:544-555, 2011.

[39] B. Bollobás and A. D. Scott. Intersections of graphs. Journal of Graph Theory, 66(4):261-282, 2011

[40] C. Borgs, J. Chayes, R. Hofstad, G. Slade, and J. Spencer. Random subgraphs of finite graphs: III. The phase transition for the n-cube. Combinatorica, 26(4):395-410, 2006.

[41] S. Nikoletseas, C. Raptopoulos, and P. Spirakis. Large independent sets in general random intersection graphs. Theororetical Computer Science, 406(3):215-224, Oct. 2008

[42] K. Rybarczyk. Constructions of independent sets in random intersection graphs. Theoretical Computer Science, 524(0):103 - 125, 2014.

[43] M. Bradonjić, A. Hagberg, N. W. Hengartner, N. Lemons, and A. G. Percus. The phase transition in inhomogeneous random intersection graphs. Arxiv e-prints, 2013. Available online at http://arxiv.org/abs/1301.7320

[44] S. Janson, T. Łuczak, and A. Ruciński. Random graphs. WileyInterscience Series on Discrete Mathematics and Optimization, 2000.

[45] M. Bradonjić and I. Saniee. Bootstrap percolation on random geometric graphs. Probability in the Engineering and Informational Sciences, 28(2):169-181, 2014.

[46] M. Bradonjić, R. Elsässer, T. Friedrich, T. Sauerwald, and A. Stauffer. Efficient broadcast on random geometric graphs. In Proc. of SODA, pages 1412-1421, 2010.

[47] P. Gupta and P. R. Kumar. Critical power for asymptotic connectivity in wireless networks. In Proc. IEEE CDC, pages 547-566, 1998.

[48] A. Goel, S. Rai, and B. Krishnamachari. Monotone properties of random geometric graphs have sharp thresholds. The Annals of Applied Probability, 15(4):2535-2552, 2005.

[49] Y. Peres, A. Sinclair, P. Sousi, and A. Stauffer. Mobile geometric graphs: detection, coverage and percolation. Probability Theory and Related Fields, 156(1-2):273-305, 2013.

[50] Q. Wang, X. Wang, and X. Lin. Mobility increases the connectivity of k-hop clustered wireless networks. In Proc. of ACM MobiCom, pp. 121-132, 2009

[51] K. Censor-Hillel, M. Ghaffari, and F. Kuhn. A new perspective on vertex connectivity. In Proc. of SODA, pages 546-561, 2014.

[52] P. Erdős and A. Rényi. On random graphs, I. Publicationes Mathematicae (Debrecen), 6:290-297, 1959.

[53] P. Erdős and A. Rényi. On the strength of connectedness of random graphs. Acta Math. Acad. Sci. Hungar, pages 261-267, 1961. 